\title{
Modelization of surface diffusion of a molecular dimer
}

\author{
A. H. Romero, ${ }^{1}$ A. M. Lacasta, ${ }^{2}$ and J. M. Sancho ${ }^{3}$ \\ ${ }^{1}$ Advanced Materials Department, IPICyT, Camino a la presa San José 2055, Codigo Postal 78216, San Luis Potosí, SLP, Mexico \\ ${ }^{2}$ Departament de Física Aplicada, Universitat Politècnica de Catalunya, Avenida Dr. Marañon 44, E-08028 Barcelona, Spain \\ ${ }^{3}$ Departament d'Estructura i Constituents de la Matèria, Facultat de Física, Universitat de Barcelona, Diagonal 647, \\ E-08028 Barcelona, Spain
}

(Received 27 January 2004; published 20 May 2004)

\begin{abstract}
A simple model for a dimer molecular diffusion on a crystalline surface, as a function of temperature, is presented. The dimer is formed by two particles coupled by a quadratic potential. The dimer diffusion is modeled by an overdamped Langevin equation in the presence of a two-dimensional periodic potential. Numerical simulation's results exhibit some dynamical properties observed, for example, in $\mathrm{Si}_{2}$ diffusion on a silicon [100] surface. They can be used to predict the value of the effective friction parameter. Comparison between our model and experimental measurements is presented.
\end{abstract}

DOI: 10.1103/PhysRevE.69.051105

PACS number(s): 05.40.-a, 66.30.Pa, 68.35.Fx, 82.56.Lz

Molecular diffusion is one of the typical examples in transport phenomena where the crossing of a potential barrier is the main physical mechanism. The way a dimer is physisorbed or chemisorbed on a surface affects its diffusive motion. Experimental studies by using scanning tunneling microscope (STM) of dimer diffusion on a crystalline surface show a very rich phenomenology [1]. The understanding of the diversity of diffusion mechanisms is a challenging problem with important implications in fields such as heteroepitaxial crystal growth, chemical etching, chemical vapor deposition, and chemical surface absorption. A molecule can explore a surface by jumping potential barriers and partially governing the absorption. Our aim here is to study the diffusive aspects of this dynamical process by means of a simple statistical model within experimental scales.

The last few years have witnessed an increasing interest in molecular diffusion due to the development of more sophisticated experimental setups which are able to follow the molecule path during diffusion [2-4]. For example, in the area of microelectronic devices there is a particular interest to understand the elementary processes involved in the homoepitaxial silicon crystal growth [5-11]. A particular case of this growth is the diffusion of small $\mathrm{Si}_{2}$ molecules on a $\mathrm{Si}$ surface. The $\mathrm{Si}[100]$ surface reconstructs in dimer rows with different characteristic lengths. Due to this surface anisotropy, dimer diffusion can adopt many different configurations. STM variable temperature experiments [8] indicate a preferential direction. The activation barrier for different dimer orientations can be estimated from experimental measurements [1,7] and it has been theoretically determined by $a b$ initio calculations $[9,11]$. Basically, there are two more favorable configurations which are parallel or perpendicular to the dimer surface reconstruction (crystal [110] orientation). At finite temperatures the deposited dimers are able to diffuse and interchange between these two principal orientations as observed by STM [1,7].

Due to the importance of the silicon diffusion, we have applied our model to this physical system. However, there are many other examples where molecule diffusion is also important, like in large molecules on metallic surfaces
$[12,13]$, nonrigid molecules on surfaces [14], in the growth of Si-Ge alloys [16], gold dimer diffusion on metallic surfaces [13], iridium dimers on iridium surfaces [15], etc. We think that these cases can also be rationalized using the same procedure we discuss below.

In order to understand the behavior of molecular diffusion many different approaches have been followed in the literature, ranging from ab initio molecular dynamics, semiempirical calculations (tight binding) to parametrized potentials, as in classical molecular dynamics. Most of them have tried to explain the details of the diffusion, starting from surface reconstruction up to molecular mobility on the reconstructed surface. Even though many results can be obtained from such calculations, it is difficult to extrapolate them to experimental time scales, which usually are of seconds. Here we propose a phenomenological approach based on the numerical simulation of the Langevin equation for a set of noninteracting dimers in the presence of a periodic potential, which mimic the surface underneath. We neglect the precise surface details and we only take into account the most relevant scales. Within this simple approach we are able to reproduce results obtained from other, but more demanding, methods and to make experimentally observable predictions.

All this phenomenology suggests, that even though the potential energy surface is rather complicated, it can be simplified by keeping only a periodic potential with two different spatial scales, $\lambda_{x}$ and $\lambda_{y}$, along the $x$ and $y$ Cartesian axes, respectively. In the case of a silicon surface, the characteristic length scales are the dimer-dimer distance on the same row and the distance between different rows. A dimer is considered as a diatomic molecule connected by a harmonic spring, with a characteristic vibrational frequency and equilibrium distance given by the ad-dimer experimental or $a b$ initio data (with a mismatch with respect to the crystalline reconstructed surface). Within this geometrical scheme the ad-dimer atoms lie close in two neighboring wells. In our particular example, the $x$-axis is defined along the [110] direction and the $y$-axis along the $[\overline{1} 10]$ direction of the silicon surface. We denote the two most probable dimer configurations as $A$ and $B$ following the notation introduced in Ref. 


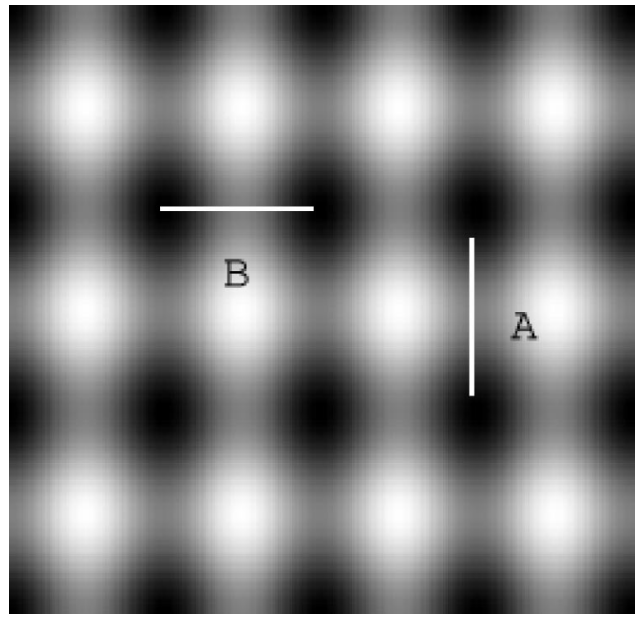

FIG. 1. Plot of the two-dimensional potential and dimer most probable configurations in dimensionless units.

[1]). Any other configuration, such as along the wells diagonal, are not very probable.

According to our previous discussion we simulate dimer diffusion on a crystalline surface by the following set of Langevin equations, in the overdamped limit:

$$
\gamma \dot{\overrightarrow{x_{k}}}=-\nabla_{k} V-\nabla_{k} U+\vec{\xi}_{k}(t),
$$

where subindex $k=1,2$ stands for each particle, and $\gamma$ is the friction parameter and $\vec{x}$ is the particle vector position. The local potential $V$ has a simple periodic form,

$$
V=V_{0} \cos \pi\left(x_{k} / \lambda_{x}+y_{k} / \lambda_{y}\right) \cos \pi\left(x_{k} / \lambda_{x}-y_{k} / \lambda_{y}\right) .
$$

This potential has maximum at $\left(n \lambda_{x}, m \lambda_{y}\right)$ and minimum at $\left[(n+1 / 2) \lambda_{x},(m+1 / 2) \lambda_{y}\right]$, with a barrier height of $V_{0}$ at the saddle points connecting two consecutive minimum. A twodimensional plot of the periodic potential is shown in Fig. 1, where the dark color represents the minima and the bright one the maximal values of the potential. The interaction potential between the dimer atoms is

$$
U=\frac{1}{2} \mu \omega^{2}\left(d_{12}-a\right)^{2},
$$

where $d_{12}$ is the instantaneous distance between the two atoms, $a$ is the equilibrium distance, $\mu=m / 2$ is the dimer reduced mass, and $\omega$ is the natural dimer vibrational frequency. The thermal noise acting on the dimer is a Gaussian and white noise with zero mean and correlation, $\left\langle\xi_{i k}(t) \xi_{j l}\left(t^{\prime}\right)\right\rangle$ $=2 \gamma K_{B} T \delta_{i j} \delta_{k l} \delta\left(t-t^{\prime}\right)$.

We can proceed now by defining a new reduced set of independent parameters, by the appropriate change of variables,

$$
\vec{x}_{k}=a \vec{r}_{k}, \quad t=\frac{\gamma a^{2}}{V_{0}} s,
$$

which makes the time and spatial variables dimensionless. Accordingly, the length scale is given in $a$ units, and the time scale controlled by the unknown friction parameter $\gamma$. The value of this parameter will be estimated from experimental time scales.
With these new definitions, the equations of motion (1) transform into

$$
\dot{\vec{r}}_{k}=-\nabla_{k} \mathcal{V}-\nabla_{k} \mathcal{U}+\vec{\eta}_{k}(t)
$$

where

$$
\begin{gathered}
\mathcal{V}=\cos \pi\left(\frac{a}{\lambda_{x}} r_{x k}+\frac{a}{\lambda_{y}} r_{y k}\right) \cos \pi\left(\frac{a}{\lambda_{x}} r_{x k}-\frac{a}{\lambda_{y}} r_{y k}\right), \\
\mathcal{U}=\frac{1}{2} k^{\prime}\left(d_{12}^{\prime}-1\right)^{2}, \quad k^{\prime}=\frac{\mu \omega^{2} a^{2}}{V_{0}},
\end{gathered}
$$

with the new distances between atoms $d_{12}^{\prime}$ $=\sqrt{\left(r_{x 1}-r_{x 2}\right)^{2}+\left(r_{y 1}-r_{y 2}\right)^{2}}$, and now the noise term has a correlation,

$$
\left\langle\eta_{i k}(s) \eta_{j l}\left(s^{\prime}\right)\right\rangle=2 \varepsilon \delta_{i j} \delta_{k l} \delta\left(s-s^{\prime}\right)
$$

with $\varepsilon=K_{B} T / V_{0}$.

The parameters of the model are $k^{\prime}, \varepsilon, a, \lambda_{x}$, and $\lambda_{y}$. Changing some of these parameters could mean just to change the nature of the substrate or the dimer.

Returning to the example which motivates this work, we take the values of the parameters from $a b$ initio or experimental data of $\mathrm{Si}_{2}$ on $\mathrm{Si}$ surface. The $\mathrm{Si}_{2}$ is parametrized by the equilibrium distance $a=2.25 \AA$ and its normal frequency $\omega=500 \mathrm{~cm}^{-1}$. Assuming that $V_{0}=0.5 \mathrm{eV}$, we get $k^{\prime}=30$. The experimental observation that the dimer has different probabilities for each configuration $[1,7]$ can be implemented in our model with a proper selection of the different spatial scales, $\lambda_{x}=2.35 \AA$ and $\lambda_{y}=3.05 \AA$, which mimics the surface anisotropy observed by the silicon ad dimer. Again, as we have pointed out before, the reconstructed silicon surface has more length scales, but from the dimer diffusion point of view only two of them are responsible for the most probable configurations. As shown below, it is sufficient to consider those length scales to calculate the diffusion properties and to obtain the dynamics. Another parameter in the simulation is the time integration step $\Delta s=0.001$. We have averaged out all our observations over a population of 1000 noninteracting dimers (and sometimes 5000) to decrease statistical errors.

Our objective here is to study the diffusive motion of the dimer and accordingly to evaluate the diffusion coefficients defined as

$$
D_{r_{i}}=\lim _{s \rightarrow \infty} \frac{\left\langle R_{i}^{2}(s)\right\rangle}{2 s},
$$

where $R_{i}(s)$ is a Cartesian component of the center of mass of the dimer.

As a preliminary check of our approach we have considered a single Si atom diffusing on a periodic potential, with the same model parameters introduced above. Our numerical results indicate that the diffusion coefficient is sensitive to the temperature (Kramers' dependence), but quite independent of the asymmetry of the potential, as expected. Only for high temperatures the diffusion coefficient start to indicate small directional difference, which constitute corrections to Kramers' prediction. 

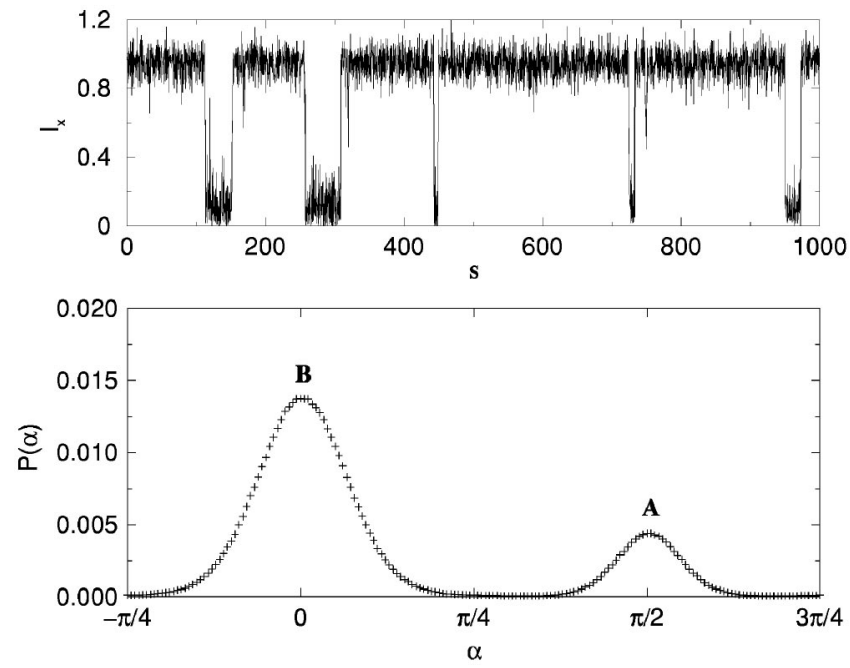

FIG. 2. (Top) Typical evolution of dimer orientation corresponding to $\epsilon=0.2, l_{x}(s)$ is the normalized dimer length as defined in the text. (Bottom) Histogram of the instantaneous dimer angle with respect to $x$ axis for $\epsilon=0.2$.

Results are much more appealing when we consider dimer diffusion. First we see that the dimer is usually in one of the two configurations of Fig. 1. Its orientation is given by the angle between the dimer and the $x$-axis, as $l_{x}(s)=\mid x_{1}(s)$ $-x_{2}(s) \mid / \lambda_{x}$. In Fig. 2, we plot a typical time evolution of $l_{x}(s)$. This figure clearly shows that only two possible configurations are probable ( $A$ and $B$ of Fig. 1). The path followed by the dimer from one configuration to the other is through an intermediate diagonal step, where the atoms are located in two contiguous diagonal wells. This diagonal intermediate configuration has a very short lifetime and is not observed in our simulations. $l_{x} \sim 1$ corresponds to configuration $B$ (dimer in horizontal position) and $l_{x} \sim 0$ to configuration $A$ (vertical position). Our choice of the parameters $\left(\lambda_{x}, \lambda_{y}, a\right)$ makes the configuration $B$ more probable than the $A$ configuration, as observed in experiment [18]. This is due to the fact that the equilibrium distance $a=2.25 \AA$ is closer to the horizontal length scale $\lambda_{x}=2.35 \AA$. This observation can be quantified by evaluating the histogram (averaged out in time and in realizations) of the instantaneous dimer angle along with the $x$-axis as shown in Fig. 2. The time average has been done taking the orientation every given number of steps to avoid angular correlations.

The energy associated with these two spatial configurations can be estimated assuming Kramers' law, and calculating the average residence time in a given state (say $A$ and $B$ ), as function of temperature (Fig. 3)

$$
\langle S\rangle_{i}=S_{0} e^{E_{i} / k_{B} T} .
$$

We have considered different values of $\epsilon$, corresponding to temperatures from $2.5 T_{\text {room }}$ to $7 T_{\text {room }}\left(T_{\text {room }}=300 \mathrm{~K}\right)$. From a numerical fitting of Eq. (10) we get the energy which is required to transit from one state to the top of the barrier $\left(E_{0}=0\right)$. We have obtained $E_{A}=-0.58 \mathrm{eV}$ and $E_{B}=-0.71 \mathrm{eV}$. The obtained difference $(\sim 0.1 \mathrm{eV})$ is of the same order as in experiment $[7,18]$.

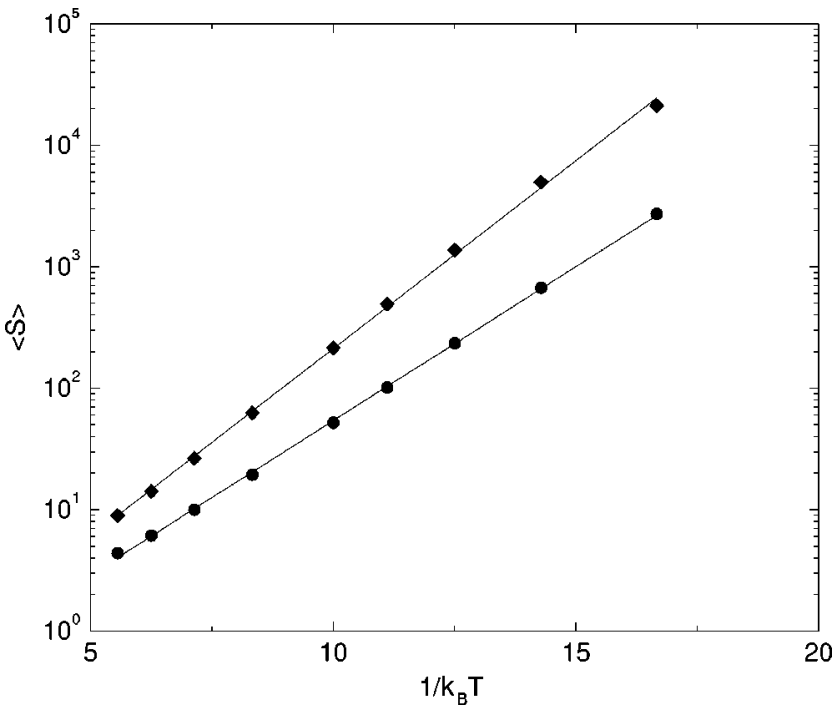

FIG. 3. Average resident dimensionless time in state $B$ (diamonds) and state $A$ (circles). $k_{B} T$ in $\mathrm{eV}$.

Another important quantity is an effective diffusion coefficient obtained for every Cartesian direction, as function of $\epsilon$. Results are presented in Fig. 4. From our model we have obtained an asymmetry given by $D_{y}>D_{x}$. This difference in diffusion coefficients can be tested experimentally since dimer diffusion asymmetries have been measured for similar configurations [19]. It is clear that a transition between configuration $A$ and $B$ or reversed, requires the same displacement change $(|\Delta x|,|\Delta y|)=\left(\lambda_{x} / 2, \lambda_{y} / 2\right)$. This indicates that we should not find any temperature dependent asymmetry when diffusion occurs. The origin of the asymmetry is a different one: it is a direct consequence of the larger stability of the dimer in configuration $B$ relative to configuration $A$. As the dimers spend most of the time in configuration $B$, the vertical motion (perpendicular to the horizontal dimer orientation) is favored and hence $D_{y}>D_{x}$. The ratio between diffusion coefficients in both directions is expected to be pro-

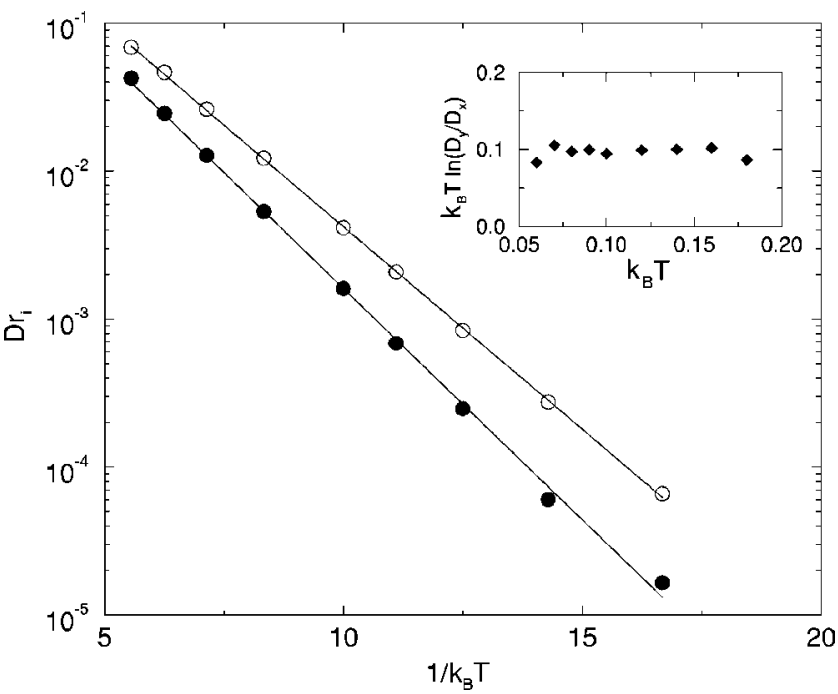

FIG. 4. Dimensionless diffusion coefficients in $x$ direction (black dots) and $y$ direction (white dots) for a $\mathrm{Si}_{2}$ dimer. $k_{B} T$ in $\mathrm{eV}$. 
portional to the ratio between the state waiting times in $B$ and A

$$
\frac{D_{y}}{D_{x}} \sim \frac{\left\langle S_{B}\right\rangle}{\left\langle S_{A}\right\rangle} \sim \exp \left(\frac{E_{A}-E_{B}}{K_{B} T}\right) .
$$

The inset of Fig. 4 shows the quantity $k_{B} T \ln \left(D_{y} / D_{x}\right)$ as a function of temperature. This value is of order $0.1 \mathrm{eV}$, in agreement with previous estimation from Fig. 3.

Finally, we can determine the unknown friction parameter $\gamma$. From Eq. (4) we see that this parameter controls the time scale transformation between an experiment ( $t$ variable) and our simulation ( $s$ variable). A characteristic experimental time scale is obtained from Ref. [17], as $T_{\text {exp }}=\nu_{o}^{-1} \sim 10^{-11} \mathrm{~s}$. From our simulation results we can also get an equivalent quantity from a numerical fitting of Eq. (10), which yields $S_{0} \sim 0.17$. Using the time scale transformation in Eq. (4) we can estimate the value of the effective friction parameter

$$
\gamma=\frac{T_{\exp } V_{0}}{S_{0} a^{2}} \sim 10^{-10} \mathrm{~kg} / \mathrm{s} .
$$

This dimensionless friction coefficient can be used to obtain an estimation of value of the unknown effective friction coefficient.

Summarizing, we have proposed a simple and general model for particle/molecular diffusion of dimers on a crystalline surface. Our model oversimplifies the real situation by only taking the relevant scales and by using experimental information as input parameters. It consists of a Langevin equation, with noise and friction terms, in the overdamped limit. The surface is described by a lattice of periodic potential wells and the dimer by particles coupled through a classical spring. We have taken, as an example, the diffusion of a $\mathrm{Si}_{2}$ dimer on a reconstructed silicon [100] surface. The different relative population in each of the two most probable configuration states observed in experiments has been incorporated in our model by considering two different spatial lengths in the $(x, y)$ directions. We analyzed the waiting time in each configuration and the diffusion coefficients as a function of temperature. We have found an anisotropy in the diffusion coefficient with respect to the directional movement. This is related to the dimer resting time in any of the $A$ or $B$ configurations. This observation is in qualitative agreement with the theoretical predictions of Ref. [9]. Our model has one free parameter to relate with the experimental time scale; by setting up this parameter we are able to estimate the effective surface friction. We contrasted the diffusion properties of the ad dimer with the atom diffusion, finding that the crystal anisotropy has more influence on the ad dimer.

We acknowledge financial support from the Dirección General de Enseñanza Superior (Spain) under project No. BFM2003-07850, and Millennium Initiative, ConacytMexico, under Grant No. W-8001. This material has been also supported by a grant from the University of California Institute for Mexico and the United States (UC MEXUS) and the Consejo Nacional de Ciencia y Tecnología de México (CONACYT).
[1] H. J. W. Zandvliet, B. Poelsema, and B. S. Swartzentruber, Phys. Today 54(7), 41 (2001).

[2] W. D. Luedtke and U. Landman, Phys. Rev. Lett. 82, 3835 (1999).

[3] M. Schunack, T. R. Linderoth, F. Rosei, E. Laegsgaard, I. Stensgaard, and F. Besenbacher, Phys. Rev. Lett. 88, 156102 (2002).

[4] M. Schulz, S. Stepanow, and S. Trimper, Europhys. Lett. 54, 424 (2001)

[5] B. Borovsky, M. Krueger, and E. Ganz, Phys. Rev. Lett. 78, 4229 (1997).

[6] G. D. Lee, C. Z. Wang, Z. Y. Lu, and K. M. Ho, Phys. Rev. Lett. 81, 5872 (1998).

[7] J. M. Carpinelli and B. S. Swatzentruber, Surf. Sci. 411, L828 (1998).

[8] Y.-W. Mo, J. Kleiner, M. B. Webb, and M. G. Lagally, Phys. Rev. Lett. 66, 1998 (1991).
[9] A. P. Smith and H. Jónsson, Phys. Rev. Lett. 77, 1326 (1996).

[10] D. R. Bowler, Phys. Rev. B 67, 115341 (2003).

[11] G. Brocks and P. J. Kelly, Phys. Rev. Lett. 76, 2362 (1996).

[12] F. Rosei, M. Schunack, Y. Naitoh, P. Jiang, A. Gourdon, E. Laegsgaard, I. Stensgaard, C. Joachim, and F. Besenbacher, Prog. Surf. Sci. 71, 95 (2003).

[13] F. Montalenti, F. Baletto, and R. Ferrando, Surf. Sci. 454, 575 (2000).

[14] C. Fusco and A. Fasolino, Thin Solid Films 428, 1 (2003).

[15] T.-Y. Fu and T. T. Tsong, Surf. Sci. 421, 1 (1999).

[16] Z.-Y. Lu, C. Z. Wang, and K. M. Ho, Surf. Sci. 506, 183 (2002).

[17] B. S. Swartzentruber, Phys. Rev. Lett. 76, 459 (1996).

[18] B. S. Swartzentruber, A. P. Smith, and H. Jónsson, Phys. Rev. Lett. 77, 2518 (1996).

[19] B. Borovsky, M. Krueger, and E. Ganz, Phys. Rev. B 59, 1598 (1999). 